\title{
Molecular Distinguishment of Trapa natans L. Varieties in Taihu Lake Region of China and Development of a RAPD-SCAR Marker for Authentication of 'Heshangling'
}

\author{
Yanmei Zhang \\ Institute of Botany, Jiangsu Province and Chinese Academy of Sciences, \\ Nanjing 210014, China \\ Xuelin Shen \\ Seed Administrative Station of Suzhou, Suzhou 215011, China
}

Xiaoqin Sun and Jia Liu

Institute of Botany, Jiangsu Province and Chinese Academy of Sciences, Nanjing 210014, China

Yifeng Xia and Xin Zou

Seed Administrative Station of Suzhou, Suzhou 215011, China

\author{
Yueyu Hang ${ }^{1}$ \\ Institute of Botany, Jiangsu Province and Chinese Academy of Sciences, \\ Nanjing 210014, China
}

Additional index words. Trapa natans, molecular distinguishment, RAPD, SCAR, Heshangling

Abstract. Water chestnut (Trapa natans $\mathbf{L}$.) is a group of annual, floating-leaved aquatic plants that serves as food and medical resources in many countries. However, the molecular method for distinguishing different $T$. natans $L$. resources is lacking. In this study, we detected genetic diversity of several chloroplast and nuclear genic or intergenic sequences in four varieties of $T$. natans and one wild type of Trapa incisa Siebold \& Zuccarini to evaluate their potential as molecular markers. Our data revealed that the three chloroplast fragments ( $r b c L, m a t K$, and $p b s A-t r n H$ ) show no sequence difference among all tested samples. Only one nucleotide substitution is detected for the nuclear ribosomal internal transcribed spacer (ITS) in the T. natans variety Shuihongling. Four nucleotide substitutions are detected for the nuclear carotenoid isomerase (CRTISO) gene in the variety Hongxiuxie. In contrast, a total of 29 polymorphic sites are detected for a Toll and interleukin-1 receptor-nucleotide binding site-leucine rich repeat (TNL) gene in the five samples, among which six are nucleotide substitutions and the rest are insertions/ deletions. The five samples could be fully distinguished from each other based on the $T N L$ gene. To specifically authenticate 'Heshangling', 33 randomly amplified polymorphic DNA (RAPD) markers were adopted to amplify genomic sequences from the five samples. A pair of sequence characterized amplified region (SCAR) primers were designed based on the results of RAPD markers, which could specifically amplify one target band from all eight individuals of 'Heshangling', but none from any individuals of other $T$. natans varieties or one $T$. incisa. Taken together, a $T N L$ sequence was provided in this study to distinguish four $T$. natans varieties and one $T$. incisa. Furthermore, a RAPDSCAR marker was developed for efficient authentication of 'Heshangling'.

Water chestnut (Trapa natans L.) is an aquatic floating herb that belongs to the family Trapaceae. It is an important aquatic economic plant, mainly distributed in the

Received for publication 28 Feb. 2018. Accepted for publication 6 May 2019.

This work was supported financially by the earmarked fund for Jiangsu Agricultural Industry Technology System to X. Shen, grant no. JATS (2018) 064.

${ }^{1}$ Corresponding author. E-mail: hangyueyu@ cnbg.net. tropical, subtropical, and temperate regions of Eurasia and Africa. In China, it is widely distributed, especially in the middle and lower reaches of the Yangtze River (Wang, 2012). The cultivation of water chestnut has a long history in China, which could be traced back to the Zhou Dynasty $\approx 3000$ years ago. The Taihu Lake area is probably the origin center of the domestication and cultivation of water chestnut in China (Hui and Cao, 2015). In the Tang Dynasty (618-907 A.D.), water caltrop was an important food for worship as prayer offerings.
Traditionally, the classification of Trapa species has largely relied on the morphological diversity; however, the complex variation of morphological traits of the genus has raised a great controversy about the species division of the Trapa genus. For example, the Trapa genus has been classified into 70 species, 30 species, 20 species, and 13 species by different studies (Cook, 1990; Kak, 1988; Tutin et al., 1968; Vassiljev, 1965). The Chinses Advanced Aquatic Plant Illustration describes 30 species in the genus, and 11 are in China (Yan, 1983). Flora Reipublicae Popularis Sinicae records 15 species of the genus, whereas Flora of China proposes that Trapa consists of only two species, $T$. natans and T. incisa (Chen et al., 2007; Wan, 2000). In the past decades, quantitative taxonomy, cell taxonomy, pollen morphology, and other methods have been used for the systematics of the genus. Taking quantitative classification study as an example, this method divides the Trapa genus in the Hubei province of China into five species and eight varieties (Xiong et al., 1985). Another study, according to the morphology of pollens, flowers, and fruits, reassigns nine previously defined Trapa species in the Zhejiang province of China to three species, namely $T$. incisa var. Sieb, Trapa bicornis Osbeck, and T. incisa (Ding et al., 1999). By measuring the size of the fruit, Wang et al. (2006) divides Trapa species in China into three groups. The horticultural classification and standard used by Peng et al. (1998) divides Trapa into Tapa quadrispinosa Roxb, Tapa bispinosa Roxb, and Tapa acornis Nakano. Therefore, the authentication of a particular Trapa species or variety has been greatly hampered by the undistinguishable morphologies and the uncertainty of classification criteria of the genus.

In recent years, the rapid development of molecular biological technology has enabled researchers to efficiently distinguish specific species or varieties with characteristic sequence features. Many nuclear genes, chloroplast genes and mitochondrial genes are used for species-level identification. Different molecular markers have also been used to study the genetic relationship and species identification of the Trapa genus. For example, several RAPD molecular markers, a nuclear gene APETALA2 (AP2), and the chloroplast gene trnL-F have proved to be capable of distinguishing $T$. natans and $T$. incisa (Jiang and Ding, 2004; Kim et al., 2010). Comparing with the interspecies differences, identification at lower taxonomic levels usually has relatively fewer genetic differences due to shorter differentiation time. The application of conserved interspecies molecular markers among lower taxonomic units usually has limitations in providing adequate genetic signals. Therefore, genes with rapid evolutionary rate are desirable for below species-level analysis. One type of well-known fast-evolving gene is plant disease resistance gene ( $R$ gene), the evolution rate of which could be several 
times faster than that of many other genes (Zhang et al., 2011).

$N B S-L R R$ genes compose the largest group of plant $R$ genes, which occupied more than $60 \%$ of known functional $R$ genes (Kourelis and van der Hoorn, 2018). Three $N B S$-LRR subclasses have been characterized, termed RESISTANCE TO POWDERY MILDEW8 (RPW8)-NBS-LRR (RNL), coiledcoil-NBS-LRR (CNL), and Toll and interleukin1 receptor-NBS-LRR (TNL) (Shao et al., 2016, 2019). These genes show distinct evolutionary pattern due to their functional difference. In a previous study, 21 different haplotypes from 39 Glycine max varieties were identified using a single $T N L$ gene Rppl (Kim, et al., 2012). Another study of Li (2014) showed that the CNL gene RPS2 can be used to identify different populations of wild mustard in China. These studies suggested that plant $R$ genes have the potential to be candidates of molecular markers below species level.

Heshangling is a local variety of $T$. natans in Wuxian, Suzhou (a city located in Taihu Lake region) and has a long cultivation history. Its trivial name comes from its semicircle fruit, with one flat side and bulging side. The shell of 'Heshangling' is relatively thin. It is well known for being juicy, crispy, sweet, and tasty. Although its fruit morphology is characteristic, it is hard to distinguish this particular variety from other water chestnut varieties cultivated in the same region before maturity. It is also difficult to distinguish commercial products made from 'Heshangling' with those from other $T$. natans varieties. As a result, development of efficient and specific molecular markers is of a great scientific and commercial significance for the protection of varieties of traditional crops.

\section{Materials and Methods}

Plant materials. Four varieties of $T$. natans commonly cultivated in the middle and lower reaches of the Yangtze River were collected from Suzhou Vegetable Research Institute. One wild type of $T$. incisa Siebold \& Zuccarini species was collected outside the Menan village of Meili town, Suzhou city (Table 1; Supplemental Fig. 1). Fresh leaves from eight individuals for each variety were collected, dried, and kept for DNA extraction.

Total DNA extraction. A total of $100 \mathrm{mg}$ of collected leaves were crushed with tissue Lyser LT (QIAGEN, Hilden, Germany). Genomic DNA was extracted using the Easy Pure Plant Genomic DNA Kit (Beijing Full Gold Biotechnology Co., Ltd., Beijing, China) according to the manufacturer's instructions. Then, the concentration and purity of DNA was detected using the nucleic acid protein detector (Eppendorf, Hauppauge, $\mathrm{NY}$ ) and saved in $-20^{\circ} \mathrm{C}$ following adjusted to $20 \mathrm{ng} / \mu \mathrm{L}$.

Identification and analysis of NBS-LRR genes from $T$. bispinosa transcriptome. The assembled unigenes for the transcriptome of T. bispinosa ( $\mathrm{Li}$ et al., 2017) were downloaded from the National Center for Biotechnology Information Web site (https://www.ncbi.nlm. nih.gov/nuccore/GEZE00000000.1/). The identification of $N B S-L R R$ genes from $T$. bispinosa transcriptome was carried out according to our previous studies with slight modifications (Shao et al., 2014, 2016; Zhang et al., 2016). Briefly, all assembled transcripts of the transcriptome were translated into protein sequences in six frames. Then, both hidden Markov model (HMM) and BLAST searches were performed against the translated proteins using the HMM profile or amino acid sequence of NB-ARC domain (Pfam no. PF00931) as a query. All hits obtained using HMM or BLAST searches were then merged together to remove redundant hits. The remaining sequences were further subjected to the online Pfam analysis (http://pfam.sanger.ac.uk/) to verify whether they indeed possessed the NBS domain, with the E-value setting to $10^{-4}$.

Phylogenetic analysis was performed for $N B S-L R R$ genes identified from $T$. bispinosa transcriptome with those from Arabidopsis thaliana using the ML method as described in our previous studies (Shao et al., 2014, 2016; Zhang et al., 2016). The NBS-LRR genes from $T$. bispinosa transcriptome were classified into $T N L, C N L$, and $R N L$ according their phylogenetic relationship to those form $A$. thaliana.

Target sequence amplification and sequence analysis. Primers for the nuclear ITS sequence and three chloroplast genes $(r b c L$, matK, and $p s b A-\operatorname{trn} H$ ) were retrieved from a previous study (China Plant BOL Group et al., 2011). Specific primers against four nuclear fragments [carotenoid isomerase (CRTISO), GEZE01020818 (RNL), GEZE01070617 (CNL), and GEZE01034532 $(T N L)$ ] were designed using the Oligo 7.0 software basing the transcriptome of T. bispinosa. The primer is synthesized by Nanjing Genscript Biotech Co., Ltd. (Nanjing, China). Polymerase chain reaction (PCR) amplification of the target genes were carried out using the following program: predegeneration for $5 \mathrm{~min}$ at $94{ }^{\circ} \mathrm{C}$; degeneration for $45 \mathrm{~s}$ at $94{ }^{\circ} \mathrm{C}$, annealing for $45 \mathrm{~s}$ at $52^{\circ} \mathrm{C}$, extension for $1 \mathrm{~min}$ at $72^{\circ} \mathrm{C}, 30$ cycles and extension $10 \mathrm{~min}$ at $72{ }^{\circ} \mathrm{C}$. The reaction system is $20 \mu \mathrm{L}$ and its components and final concentrations are as follows: DNA template $(20 \mathrm{ng} / \mu \mathrm{L}) 1.0 \mu \mathrm{L}, 2 \times$ Reaction Mix (including $20 \mathrm{~mm}$ Tris- $\mathrm{HCl}, 100 \mathrm{~mm} \mathrm{KCl}, 33 \mathrm{~mm}$ $\mathrm{MgCl} 2,4400 \mu \mathrm{M}$ dNTPs, bromine blue) 10.0 $\mu \mathrm{L}$, upstream and downstream primers $(10 \mathrm{~mm}) 1.0 \mu \mathrm{L}$ each, taq DNA polymerase $(2.5 \mathrm{U} / \mu \mathrm{L}) 0.4 \mu \mathrm{L}$, and finally double distilled water up to $20 \mu \mathrm{L}$. The PCR reaction is carried out in Biometra (Göttingen, Germany) T1 PCR instrument. PCR products were examined electrophoretically using $1.0 \%$ agarose gels under $80 \mathrm{~V}$ for $0.5 \mathrm{~h}$ and visualized via gel imaging system. Bidirectional sequencing of purified PCR products was completed by Beijing Genomics Institute (Beijing, China) using the amplification primers. The sequencing results were processed via Sequencher 4.5 to remove lowmass sequences and subjected to MEGA6 for further analysis.

Screening of RAPD primers. DNA samples pooled by eight individuals for the four varieties of $T$. natans and T. incisa were used as templates for PCR. RAPD primers were retrieved from a previous study (Jiang and Ding, 2004; Kim et al., 2010) and synthesized by the Nanjing Genscript Biotech Co., Ltd. PCR analysis was performed as described

Table 1. Plant materials used in this study.

\begin{tabular}{|c|c|c|c|}
\hline Plant name & Species name & Variety type & Main morphological description \\
\hline Hongxiuxie & T. natans & Local cultivar, Chaohu, Anhui & $\begin{array}{l}\text { Leaf blade deltoid-rhombic; 2-horned, } \\
\text { horns horizontal; peel amaranth, thin; } \\
\text { fruit tender, soft quality }\end{array}$ \\
\hline Shuihongling & T. natans & Local cultivar, Suzhou, Jiangsu & $\begin{array}{l}\text { Leaf blade deltoid-rhombic, turquoise, } \\
\text { petiole amaranth; fruit bright red, } \\
\text { 4-horned, shell thin and fruit crispy }\end{array}$ \\
\hline Yeling & Trapa incisa & Wild variety, Changshu, Jiangsu & $\begin{array}{l}\text { Leaf blade rhombic-triangular; fruit narrowly } \\
\text { rhombic, 4-horned, horns conic; shell } \\
\text { thin, fruit glutinous }\end{array}$ \\
\hline
\end{tabular}


previously. The PCR products were examined by $2.0 \%$ agarose gel electrophoresis under $90 \mathrm{~V}$ for $1 \mathrm{~h}$ and visualized via gel imaging system.

Design and validation of a RAPD-SCAR marker. A band specific to 'Heshangling' was excised and extracted from the gel. The obtained DNA fragment was cloned into pMD19-T vector and transformed into Escherichia coli DH5 $\alpha$ competent cells (Takara Biomedical Technology (Beijing) Co., Ltd., Beijing, China). Plasmids containing target sequence were extracted from the transformed bacterial cells and sequenced by Nanjing Genscript Biotech Co. A pair of specific SCAR primers targeting the sequence of the cloned band was designed. The SCAR primers were used to validate specificity for the 40 individuals of the four varieties of $T$. natans and $T$. incisa by PCR using a procedure the same as that described previously.

\section{Results}

Amplification and sequence analysis of conserved nuclear and chloroplast genes. Three chloroplast markers ( $\mathrm{rbcl}$, matK, and pbsA-trnH) and two nuclear marker (ITS and CRTISO) genes were successfully amplified from the four varieties of $T$. natans and $T$. incisa. The obtained PCR products were subjected to direct sequence. The results showed that the obtained sequences are 695 $867,354,788-789$, and 2186 in length (Table 2), respectively. Analysis of these sequences revealed that the three chloroplast genes showed no sequence difference among the four varieties of $T$. natans and one of $T$. incisa. In contrast, the two nuclear genes show different extents of sequence diversity, with only one nucleotide substitution observed for ITS sequence isolating 'Shuihongling' from the other four samples, and four polymorphic sites detected for CRTISO to 'Hongxiuxie' separate it from the other four samples (Table 3).

Amplification and sequence analysis of $N B S-L R R$ genes. The transcriptome of $T$. bispinosa was subjected to analysis for identification of $N B S-L R R$ genes based on the previously published protocol (Shao et al., 2014, 2016; Zhang et al., 2016). A total of 71 of 73,284 assembled unigenes were found to encode NBS-LRR genes (Supplemental Table 1). After removing unigenes encoding a partial NBS domain (shorter than two-thirds of the full length), the remaining 53 sequences were subjected to phylogenetic analysis with the NBS-LRR genes from $A$. thaliana. The phylogeny (Supplemental Fig. 2) showed that the $53 N B S-L R R$ transcripts from $T$. natans could be assigned to three $N B S-L R R$ subclasses $(R N L, C N L$, and $T N L$ ) basing their relationship to $N B S-L R R$ genes from $A$. thaliana. There are 3,22 , and
28 genes in $R N L, C N L$, and $T N L$ subclasses, respectively.

One unigene from each class (GEZE01020818 from $R N L$, GEZE01070617 from $C N L$, and GEZE01034532 from $T N L$ ) were selected to design specific primers. The primers were designed to target the LRR domain of each gene to obtain maximal sequence diversity, although partial NBS domain was also covered by the primers for the $T N L$ gene (Supplemental Table 2). All of the three primer pairs successfully amplified orthologous genes from the four $T$. natans varieties and $T$. incisa. Sequence analysis revealed that there was no difference for the selected $R N L$ and $C N L$ genes among the five samples. In contrast, the $T N L$ sequences from the five samples have 29 detected polymorphism sites in the coding region, of which six are nucleotide substitutions, 23 are insertions/ deletions. 'Heshangling' has a four-base deletion from the position 1071 to 1074 , whereas $T$. incisa has a 19 base pair (bp) insertion at the position of $\approx 926$ to 944 . These $T N L$ gene fragments amplified from the five samples are all different from each other; therefore, may serve as a molecular marker to distinguish different $T$. natans varieties and $T$. incisa.

RAPD molecular marker screening for Heshangling varieties. PCR amplification was carried out on four varieties of T. natans and $T$. incisa with 33 RAPD primers. The

Table 2. Primers used in this study.

\begin{tabular}{|c|c|c|}
\hline Primers & Primer sequence & Amplified sequence length \\
\hline $\operatorname{mat} K-\mathrm{F}$ & CGTACAGTACTTTTGTGTTTACGA & 867 \\
\hline $\operatorname{mat} K-\mathrm{R}$ & ACCCAGTCCATCTGGAAATCTTGGTTC & \\
\hline psbA-trnH-R & CGCGCATGGTGGATTCACAATCC & \\
\hline ITS-F & AACAAGGTTTCCGTAGGTGA & $788-790$ \\
\hline ITS-R & TGAGGACGCTTCTCCAGAC & \\
\hline GEZE01034532-R & CTTACTAGCTTCAAATGCACCA & \\
\hline GEZE01070617-F & CTGCCTTACTACCTCAAGTCG & 816 \\
\hline GEZE01070617-R & GTCAACTTCCCAATACCССТC & \\
\hline GEZE01020818-F & TCTGATCGACCATTCAATGCC & 1373 \\
\hline GEZE01020818-R & CCAGAGATCATTGCACCGAGA & \\
\hline
\end{tabular}

Table 3. Variable sites of internal transcribed spacer (ITS), carotenoid isomerase (CRTISO), and Toll and interleukin-1 receptor-nucleotide binding site-leucine rich repeat (TNL) genes in four varieties of Trapa natans and one Traps incisa.

\begin{tabular}{|c|c|c|c|c|c|c|c|c|c|c|c|c|c|c|c|c|c|c|}
\hline \multirow[b]{2}{*}{ Plant name } & \multirow{2}{*}{$\frac{I T S}{706}$} & \multicolumn{5}{|c|}{ CRTISO } & \multicolumn{12}{|c|}{$T N L$} \\
\hline & & 618 & 1756 & 1846 & & 2019 & 441 & 516 & 699 & 851 & 916 & 918 & 926 & 927 & 928 & 929 & 930 & 931 \\
\hline Laowuling & $\mathrm{C}$ & $\mathrm{T}$ & $\mathrm{A}$ & $\mathrm{G}$ & & $\mathrm{A}$ & $\mathrm{G}$ & $\mathrm{G}$ & $\mathrm{G}$ & $\mathrm{C}$ & $\mathrm{G}$ & $\mathrm{T}$ & - & - & - & - & - & - \\
\hline Hongxiuxie & $\mathrm{C}$ & $\mathrm{C}$ & G & A & & G & A & $\mathrm{G}$ & $\mathrm{G}$ & G & $\mathrm{G}$ & $\mathrm{T}$ & - & - & - & - & - & - \\
\hline Shuihongling & A & $\mathrm{T}$ & A & G & & A & G & $\mathrm{T}$ & G & $\mathrm{C}$ & G & $\mathrm{G}$ & - & - & - & - & - & - \\
\hline Heshangling & $\mathrm{C}$ & $\mathrm{T}$ & A & $\mathrm{G}$ & & A & A & G & G & G & G & $\mathrm{T}$ & - & - & - & - & - & - \\
\hline \multirow[t]{2}{*}{ Yeling } & $\mathrm{C}$ & $\mathrm{T}$ & A & G & & A & G & G & $\mathrm{C}$ & G & G & $\mathrm{T}$ & G & A & A & G & $\mathrm{C}$ & A \\
\hline & \multicolumn{18}{|c|}{$T N L$} \\
\hline Plant name & 932 & 933 & 934 & 935 & 936 & 937 & 938 & 939 & 940 & 941 & 942 & 943 & 944 & 1071 & 1072 & 1073 & & 1074 \\
\hline Laowuling & - & - & - & - & - & - & - & - & - & - & - & - & - & $\mathrm{G}$ & $\mathrm{T}$ & $\mathrm{G}$ & & $\mathrm{T}$ \\
\hline Hongxiuxie & - & - & - & - & - & - & - & - & - & - & - & - & - & $\mathrm{G}$ & $\mathrm{T}$ & $\mathrm{G}$ & & $\mathrm{T}$ \\
\hline Shuihongling & - & - & - & - & - & - & - & - & - & - & - & - & - & G & $\mathrm{T}$ & G & & $\mathrm{T}$ \\
\hline Heshangling & - & - & - & - & - & - & - & - & - & - & - & - & - & - & - & - & & - \\
\hline Yeling & A & $\mathrm{C}$ & A & G & G & $\mathrm{T}$ & G & $\mathrm{T}$ & A & $\mathrm{T}$ & $\mathrm{T}$ & $\mathrm{C}$ & $\mathrm{C}$ & G & $\mathrm{T}$ & G & & $\mathrm{T}$ \\
\hline
\end{tabular}

"_" indicates base deletions. 


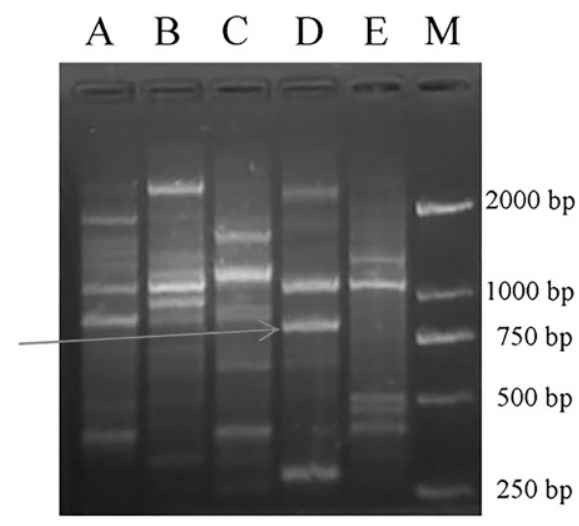

Fig. 1. Amplification profiles of four varieties of Trapa natans and one Trapa incisa using RAPD26 marker. Lanes A to E in order are 'Laowuling', 'Hongxiuxie', 'Shuihongling', 'Heshangling', and 'Yeling'. M, DL2000 DNA marker; the arrow represents a specific band of 'Heshangling'. $b p=$ base pairs.

ATTGCTTCGATACGCGTCTTGCAAACCTTATAGGCCACCATCGCACGATCAA CCATCGGAATTAGCGCGTCGATATCTGGCTCTTGTTGCGTCCTCAAGGTTTC TGCTATTTTTTTCAGCGTTGCATAGTTTTCTTTAAAAGAATCAGACTCTGCG TTCATATTATTCTCCAACTTTTAAAAATTAGTAATTCACTGCCGGTTTAACTT TGATATCGCCATCCCTAAATTCGATATTCAGAAGTTCAGCTTGCTTCGCTTGT TCTGCCGTGACGACCGGTTTACCACTGAGATCGCGGACAAGCGCAAAGCC TCGTCGTAAGGTCGCCTTGGGGCCTATGCCTAGTATCTCCCTGGCCAAGGA CTCGATTTGAATCGCGGCATCGTCAATTTTCTGTTGTCCCAACTCAGTTACT CTCCGCAAGTAAAACTCAATTTCCCGTTCGGCGTCTAATATTTGGATGTCGG CAAGGAAGGTCACTTTTTCCCATTCTTTATCGGCCAATTTTTCTGCGTCCGC CAAAACCGAAAATGATTTTGTCTCGATCCAGCGCATCCCGTGTTCGAGCCT GTtTrCGGCGGTGGaCAGGAGCATCTGAGCAGCCGGTtGTAGGCCAGCCG CAAGTTGTTCGATTTCATTATCCACCAGGTTTAATTGCTCAGAAACCTGCGT CTGGATTTTTCCGATAAAGATCCCACACGCCTTTTCAGCACTCGATAACTGC TTTTCGGTATGTAACAAGAATTCCTGAAAATGCGCCTCGGCCGTCGTCGCA TTGCTGCAAATGACATCCCTGATGTGCCCGATGACTTTTGATGGCGTATCGA AGCAATC

Fig. 2. The nucleotide sequence of 'Heshangling'specific fragment amplified using primer designed sequence characterized amplified region primers. RAPD26. The underlined sequences are

template of each DNA sample is composed of eight individuals. The products amplified from 'Heshangling' by the primer RAPD26 (5'-GAGAGAGAGAGAGAGAYT-3') showed a unique band with a size $\approx 800 \mathrm{bp}$ in addition to other bands amplified from all varieties (Fig. 1). This 'Heshangling'-specific fragment was then cloned and sequenced. The unique band finally turned out to be a fragment of $834 \mathrm{bp}$ in length; the nucleotide sequence is shown in Fig. 2.

Design and validation of a pair of RAPD$S C A R$ primers for specific identification of 'Heshangling'. A pair of SCAR primers is designed to target the 'Heshangling'-specific fragment (as shown in Fig. 2): SCAR-F (5'CTCTAACCGATTCTCACCTCC-3') and SCAR-R (5' - TATAGGCTTTTTATCAA TCGTA-3'), corresponding to the 419-444 and the 713-736 positions. PCR amplification was performed on 40 individuals from four varieties of $T$. natans and $T$. incisa by the SCAR primers. The result showed that a specific band was amplified from all eight individuals of 'Heshangling', which is in accordance with its expected size of $318 \mathrm{bp}$. In contrast, no bands were amplified from any individuals from the remaining three varieties of $T$. natans or $T$. incisa (Fig. 3).

\section{Discussion}

The morphological variation of Trapa genus is extremely complicated in the number of angles, color of peel, and the size of fruit. Investigators around the world have a great controversy regarding delimitation of Trapa species. Some proposed that there are dozens of Trapa species, and others combine them into one (Cook, 1990; Kak, 1988; Tutin et al., 1968; Vassiljev, 1965; Yan, 1983). In our country, Flora of China combined the 15 Trapa species/varieties recorded in Flora

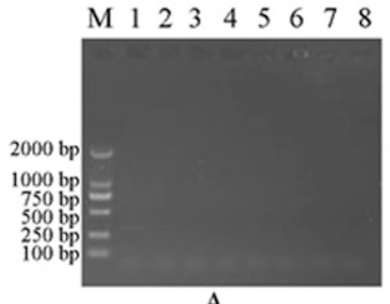

A

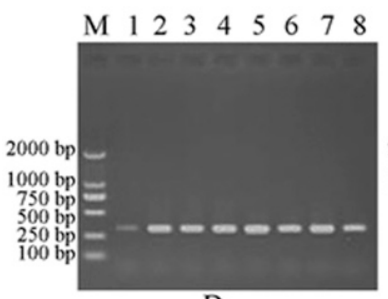

D

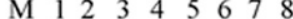

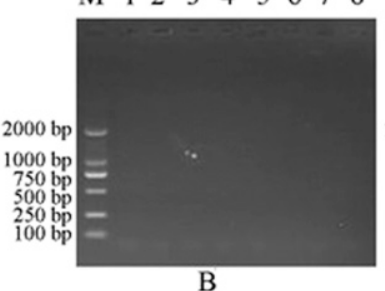

B
M 12234456678

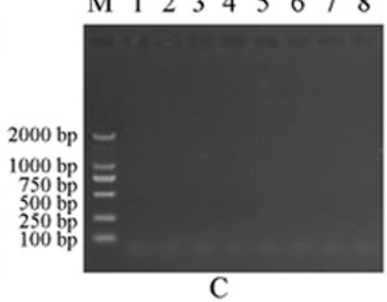

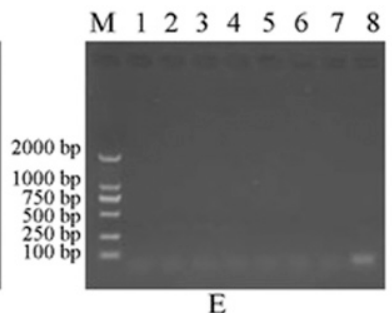

Fig. 3. Verification of the 'Heshangling'-specific randomly amplified polymorphic DNA-sequence characterized amplified region by polymerase chain reaction (PCR) in eight individuals from each of four varieties of Trapa natans and one Trapa incisa. A to E are PCR results from eight individuals of 'Laowuling', 'Hongxiuxie', 'Shuihongling', 'Heshangling', and 'Yeling', respectively. M = DL2000 DNA marker. Lanes 1 to 8 are PCR products from eight individuals. $\mathrm{bp}=$ base pairs.
Reipublicae Popularis Sinicae into two different species, $T$. natans and $T$. incisa. It seems to indicate that researchers are uncertain about the classification inside the genus Trapa, and the previous taxonomic algorithm based on morphology does not work well. China has a long history in Trapa cultivation, and Trapa is widely distributed in the middle and lower reaches of the Yangtze River. This study explored molecular markers that potentially distinguish different $T$. natans resources.

With the rapid development of sequencing technology, the molecular method has been frequently used for phylogenetic analysis and barcoding of plants. The chloroplast genes and the nuclear ITS sequence have been widely used as barcoding markers at different taxonomic levels (CBOL Plant Working Group, 2009). Previous studies have shown that the chloroplast DNA sequences are more conservative than the nuclear ITS region. They usually could distinguish taxa at or above genus level, but may not suit for the classification among closely related species within the same genus ( $\mathrm{Li}$ et al., 2018). By comparison, the ITS fits better at species-level resolution than chloroplast genes. In the present study, none of the three chloroplast markers is capable of distinguishing varieties of T. natans. ITS is not much better, showing only one single nucleotide polymorphism. As for the other chosen nuclear marker CRTISO, only one variety could be identified based on the four nucleotide substitutions.

To find more effective molecular markers, we examined one of the most rapid-evolving plant gene families, NBS-LRR genes. The $N B S$ - $L R R$ gene family is composed of hundreds of members with highly diverged sequences due to their active evolutionary patterns (Shao et al., 2016). According to the results of previous studies (Kim et al., 2012; $\mathrm{Li}, 2014)$, some $N B S-L R R$ genes could be highly variable even among individuals within the same species. Inspired by these findings, this study selected three $N B S-L R R$ genes to evaluate their sequence diversity among the four varieties of $T$. natans and $T$. incisa. Two criteria were used to select candidate $N B S-L R R$ genes for amplification in this study. First, the assembled transcript of a selected gene has a relatively long coding region and covers the LRR domain. The LRR domain is the most variable region of $N B S$ - $L R R$ genes. This criterion would help us to amplify a relatively long sequence with high polymorphism. Second, the selected gene has no detected paralogs with high sequence similarity. This criterion would avoid nonspecific amplification.

Although no sequence differences were observed for the two of them, the selected $T N L$ gene showed high sequence diversity among the five samples. Polymorphic sites of both nucleotide substitutions and insertions/ deletions were observed. Nearly all polymorphic sites were detected in the LRRencoding region, whereas none of them was 
detected in the amplified partial NBS domain region. The higher diversity of the $T N L$ gene than that of the $C N L$ and the $R N L$ genes may due to the different evolutionary pattern among the three NBS-LRR subclasses (Shao et al., 2016). $R N L$ is the most conserved NBS-LRR subclass and evolves slower than the other two subclasses due to its specific function in signal transduction. $C N L$ and $T N L$ genes are usually involved in pathogen detection and therefore show fast evolutionary rate to cope with the rapidly evolving pathogens (Shao et al., 2016). Furthermore, several studies have reported that $T N L$ genes evolve faster than $C N L$ genes; we speculate that this may be partially because of its ancient intron-rich structure compared with the intron-less structure of $C N L$ genes (Shao et al., 2016; Zhang et al., 2011), and introns are much less subject to evolutionary constraints so that allowed for higher sequence diversity. Overall, the results from our study suggested that the $T N L$ gene amplified in this study may serve as a candidate molecular marker for T. natans and T. incisa.

Although the $T N L$ gene is capable of distinguishing the tested four varieties of $T$. natans and T. incisa effectively, this method requires a professional background for molecular experiments and data analysis, as well as understanding of the theory of evolution, which might not be so appropriate in practical application. RAPD markers are DNA fragments obtained by PCR amplification of random segments from the genomic DNA. The SCAR marker is derived from RAPD that aims to specifically and efficiently amplify target bands from specified species or varieties. In the present study, a RAPD-SCAR marker for specific authentication of 'Heshangling' has been developed. Using this method, 'Heshangling' can be quickly and intuitively identified with an efficiency of $100 \%$.

Taken together, we developed two different approaches to distinguish four varieties of $T$. natans and T. incisa. The sequence character of the $T N L$ gene could distinguish all four varieties of $T$. natans and $T$. incisa simultaneously, whereas the RAPD-SCAR marker could specifically authenticate 'Heshangling'. Our study may serve as a foundation for further application of molecular markers to identify and protect the high quality of local Trapa resources, as well as commercialized products of Trapa.

\section{Literature Cited}

CBOL Plant Working Group. 2009. A DNA barcode for land plants. Proc. Natl. Acad. Sci. USA 106(31):12794-12797.

Chen, C., B.Y. Ding, and A.M. Funston. 2007. Trapaceae, 290-291. In: Z.Y. Wu and P.H. Raven (eds.). Fl. Science Press, Bejing; Missouri Botanical Garden, St. Louis.

China Plant BOL Group, D.Z. Li, L. M. Gao, H.T. Li, H. Wang, X.J. G, J.Q. Liu, Z.D. Chen, S.L. Zhou, S.L. Chen, Y.B. Yang, C.X. Fu, C.X Zeng, H.F. Yan, Y.J. Zhu, Y.S. Sun, S.Y. Chen, L. Zhao, K. Wang, T. Yang, and G.W. Duan. 2011. Comparative analysis of a large dataset indicates that internal transcribed spacer (ITS) should be incorporated into the core barcode for seed plants. Proc. Natl. Acad. Sci. USA 108(49): 19641-19646.

Cook, C.D.K. 1990. Aquatic plant book. SPB Academic publishing, The Hague, The Netherlands.

Ding, B.Y., T. Huang, W.M. Jiang, L.A. Zhao, and C.Z. Zheng. 1999. The seedling morphology of Trapa and its systematic significance. J. Zhejiang Univ. (Sci. Ed.). 26(3):92-98.

Hui, F.P. and Y. Cao. 2015. Water caltrop cultivation around Taihu lake in the Ming-Qing dynasties. Agr. Hist. China. (5):24-33.

Jiang, W.M. and B.Y. Ding. 2004. Genetic relationship among Trapa species assessed by RAPD markers. J. Zhejiang Univ. (Agr. \& Life Sci.). 30(2):191-196.

Kak, A.M. 1988. Aquatic and wetland vegetation of western Himalayas. J. Econ. Taxon. Bot. 12(2):447-451.

Kim, C., H.R. Na, and H.K. Choi. 2010. Molecular genotyping of Trapa bispinosa and T. japonica (Trapaceae) based on nuclear AP2 and chloroplast DNA trnL-F region. Amer. J. Bot. 97(12):E149-E152.

Kim, K.S., J.R. Unfried, D.L. Hyten, R.D. Frederick, G.L. Hartman, R.L. Nelson, Q. Song, and B.W Diers. 2012. Molecular mapping of soybean rust resistance in soybean accession PI 561356 and SNP haplotype analysis of the Rppl region in diverse germplasm. Theor. Appl. Genet. 125(6):1339-1352.

Kourelis, J. and R.A.L. van der Hoorn. 2018. Defended to the nines: 25 years of resistance gene cloning identifies nine mechanisms for $\mathrm{R}$ protein function. Plant Cell 30(2):285-299.

Li, J., Y. Yang, S. Yang, Z. Zhang, S. Chen, C. Zhong, R. Zhou, and S. Shi. 2017. Comparative transcriptome analyses of a mangrove tree Sonneratia caseolaris and its non-mangrove relatives, Trapa bispinosa and Duabanga grandiflora. Mar. Genom. 31:13-15.

Li, S., X. Qian, Z. Zheng, M. Shi, X. Chang, X. Li, J. Liu, T. Tu, and D. Zhang. 2018. DNA barcoding the flowering plants from the tropical coral islands of Xisha (China). Ecol. Evol. 8(21):10587-10593.
Li, Y. 2014. Genetic diversity and genetic structure of wild Brassica juncea in China. Nanjing Agr. Univ., Nanjing, China, M.S. Thesis.

Peng, J., Q.D. Kong, W.D. Ke, and Y.M. Liu. 1998 Horticultural classification of the germplasm resources of water caltrap (Trapa spp.). Adv. Hort. 2:645-649.

Shao, Z.Q., J.Y. Xue, P. Wu, Y.M. Zhang, Y. Wu, Y.Y. Hang, B. Wang, and J.Q. Chen. 2016. Large-scale analyses of angiosperm nucleotide-binding site-leucine-rich repeat genes reveal three anciently diverged classes with distinct evolutionary patterns. Plant Physiol. 170(4):2095-2109.

Shao, Z.Q., J.Y. Xue, Q. Wang, B. Wang, and J.Q. Chen. 2019. Revisiting the origin of plant NBS-LRR genes. Trends Plant Sci. 24(1):912.

Shao, Z.Q., Y.M. Zhang, Y.Y. Hang, J.Y. Xue, G.C. Zhou, P. Wu, X.Y. Wu, X.Z. Wu, Q. Wang, B. Wang, and J.Q. Wang. 2014. Longterm evolution of nucleotide-binding siteleucine-rich repeat genes: Understanding gained from and beyond the legume family. Plant Physiol. 166(1):217-234.

Tutin, T.G., V.H. Heywood, N.A. Burges, D.M. Moore, D.H. Valentine, S.M. Walters, D.A. Webb, P.W. Ball, A.O. Chater, and I.K. Ferguson. 1968. Fl. Europaea, Vol. 2: Rosaceae to Umbelliferae. Cambridge University Press, Cambridge.

Vassiljev, V. 1965. Species novae Africanicae generis Trapa L. Nov. Sist. Vyss. Rast. (Leningrad) 1965:175-179

Wan, W. 2000. Trapaceae, p. 1-26. In: C. Chen (ed.). Fl. Reipubl. Popularis Sin. Science Press, Beijing.

Wang, Q. 2012. Fruits of Hemitrapa (Trapaceae) from the Miocene of eastern China, their correlation with Sporotrapoidites erdtmanii pollen and paleobiogeographic implications. J. Paleontol. 86(1):156-166.

Wang, Y.F., B.Y. Ding, R.Y. Hui, and M.L. Jin. 2006. Analysis of morphological plasticity of Trapa from China and its taxonomic signification. J. Zhejiang Univ. (Sci. Ed.). 33(5):567572.

Xiong, Z.T., H.Q. Wang, and X.Z. Sun. 1985 Numerical taxonomic studies in Trapaceae in Hubei (I). J. Plant Sci. 3(1):45-53.

Yan, S.Z. 1983. Chinses advanced aquatic plant illustration. Science Press, Beijing, China.

Zhang, X.H., Y. Feng, H. Cheng, D. Tina, S. Yang, and J.Q. Chen. 2011. Relative evolutionary rates of NBS-encoding genes revealed by soybean segmental duplication. Mol. Genet. Genomics 285(1):79-90.

Zhang, Y.M., Z.Q. Shao, Q. Wang, Y.Y. Hang, J.Y. Xue, B. Wang, and J.Q. Wang. 2016. Uncovering the dynamic evolution of nucleotidebinding site-leucine-rich repeat (NBS-LRR) genes in Brassicaceae. J. Integr. Plant Biol. 58(2):165-177. 


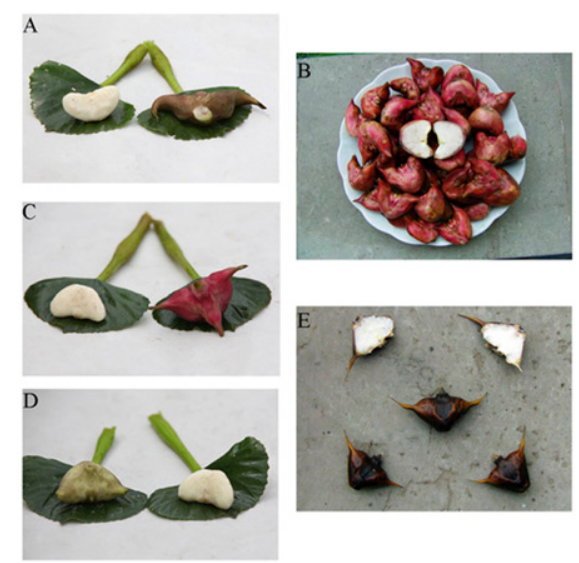

Supplemental Fig. 1. The morphological features of four varieties of Trapa natans and one wild type of Trapa incisa in this study. A to E in order are 'Laowuling', 'Hongxiuxie', 'Shuihongling', 'Heshangling', and 'Yeling'. 


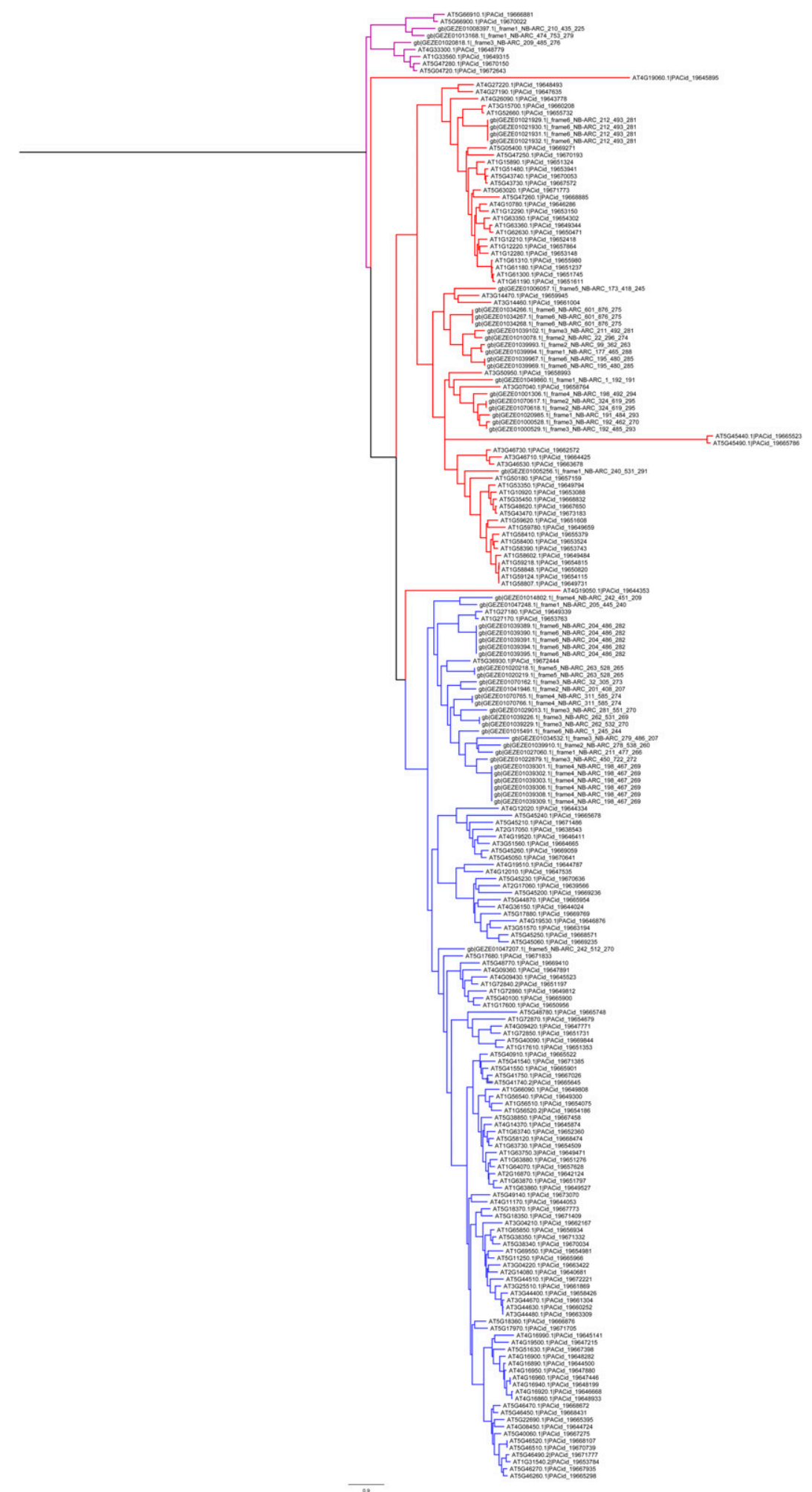

Supplemental Fig. 2. Phylogeny of Nucleotide binding site-leucine rich repeat (NBS-LRR) genes from Trapa bispinosa and Arabidopsis thaliana. 
Supplemental Table 1. Nucleotide binding site-leucine rich repeat (NBS-LRR) genes identified from Trapa bispinosa transcriptome.

\begin{tabular}{|c|c|c|}
\hline Number & Gene ID & $N B S-L R R$ subclasses \\
\hline 1 & GEZE01013168.1 & $R N L$ \\
\hline 2 & GEZE01020818.1 & $R N L$ \\
\hline 3 & GEZE01008397.1 & $R N L$ \\
\hline 4 & GEZE01000528.1 & $C N L$ \\
\hline 5 & GEZE01000529.1 & $C N L$ \\
\hline 6 & GEZE01070617.1 & $C N L$ \\
\hline 7 & GEZE01070618.1 & $C N L$ \\
\hline 8 & GEZE01001306.1 & $C N L$ \\
\hline 9 & GEZE01020985.1 & $C N L$ \\
\hline 10 & GEZE01005256.1 & $C N L$ \\
\hline 11 & GEZE01039994.1 & $C N L$ \\
\hline 12 & GEZE01039967.1 & $C N L$ \\
\hline 13 & GEZE01039969.1 & $C N L$ \\
\hline 14 & GEZE01021929.1 & $C N L$ \\
\hline 15 & GEZE01021930.1 & $C N L$ \\
\hline 16 & GEZE01021931.1 & $C N L$ \\
\hline 17 & GEZE01021932.1 & $C N L$ \\
\hline 18 & GEZE01039102.1 & $C N L$ \\
\hline 19 & GEZE01034266.1 & $C N L$ \\
\hline 20 & GEZE01034267.1 & $C N L$ \\
\hline 21 & GEZE01034268.1 & $C N L$ \\
\hline 22 & GEZE01010078.1 & $C N L$ \\
\hline 23 & GEZE01039993.1 & $C N L$ \\
\hline 24 & GEZE01006057.1 & $C N L$ \\
\hline 25 & GEZE01049860.1 & $C N L$ \\
\hline 26 & GEZE01039389.1 & $T N L$ \\
\hline 27 & GEZE01039390.1 & $T N L$ \\
\hline 28 & GEZE01039391.1 & $T N L$ \\
\hline 29 & GEZE01039394.1 & $T N L$ \\
\hline 30 & GEZE01039395.1 & $T N L$ \\
\hline 31 & GEZE01070765.1 & $T N L$ \\
\hline 32 & GEZE01070766.1 & $T N L$ \\
\hline 33 & GEZE01070162.1 & $T N L$ \\
\hline 34 & GEZE01022879.1 & $T N L$ \\
\hline 35 & GEZE01029013.1 & $T N L$ \\
\hline 36 & GEZE01039229.1 & $T N L$ \\
\hline 37 & GEZE01047207.1 & $T N L$ \\
\hline 38 & GEZE01039226.1 & $T N L$ \\
\hline 39 & GEZE01039301.1 & $T N L$ \\
\hline 40 & GEZE01039302.1 & $T N L$ \\
\hline 41 & GEZE01039303.1 & $T N L$ \\
\hline 42 & GEZE01039306.1 & $T N L$ \\
\hline 43 & GEZE01039308.1 & $T N L$ \\
\hline 44 & GEZE01039309.1 & $T N L$ \\
\hline 45 & GEZE01027060.1 & $T N L$ \\
\hline 46 & GEZE01020218.1 & $T N L$ \\
\hline 47 & GEZE01020219.1 & $T N L$ \\
\hline 48 & GEZE01039910.1 & $T N L$ \\
\hline 49 & GEZE01015491.1 & $T N L$ \\
\hline 50 & GEZE01047248.1 & $T N L$ \\
\hline 51 & GEZE01014802.1 & $T N L$ \\
\hline 52 & GEZE01034532.1 & $T N L$ \\
\hline 53 & GEZE01041946.1 & $T N L$ \\
\hline 54 & GEZE01020819.1 & $R N L$ \\
\hline 55 & GEZE01005764.1 & $R N L$ \\
\hline 56 & GEZE01005765.1 & $R N L$ \\
\hline 57 & GEZE01004861.1 & $C N L$ \\
\hline 58 & GEZE01039103.1 & $C N L$ \\
\hline 59 & GEZE01012995.1 & $C N L$ \\
\hline 60 & GEZE01006173.1 & $C N L$ \\
\hline 61 & GEZE01056022.1 & $C N L$ \\
\hline 62 & GEZE01006569.1 & $C N L$ \\
\hline 63 & GEZE01039970.1 & $C N L$ \\
\hline 64 & GEZE01064925.1 & $C N L$ \\
\hline 65 & GEZE01002219.1 & $C N L$ \\
\hline 66 & GEZE01030308.1 & $C N L$ \\
\hline 67 & GEZE01039968.1 & $C N L$ \\
\hline 68 & GEZE01039227.1 & $T N L$ \\
\hline 69 & GEZE01071760.1 & $T N L$ \\
\hline 70 & GEZE01039909.1 & $T N L$ \\
\hline 71 & GEZE01007953.1 & Undetermined \\
\hline
\end{tabular}

Note: Genes indicated in gray are not included in phylogenetic analysis due to short nucleotide binding site domain. $R N L=$ RESISTANCE TO POWDERY MILDEW8 (RPW8)-NBS-LRR; CNL = coiled-coil-NBS-LRR; $T N L=$ Toll and interleukin-1 receptor-nucleotide binding site-leucine rich repeat. 
Supplemental Table 2. Amplification regions of the Toll and interleukin-1 receptor-nucleotide binding siteleucine rich repeat (TNL), coiled-coil-NBS-LRR (CNL), and RESISTANCE TO POWDERY MILDEW8 (RPW8)-NBS-LRR (RNL) genes.

\begin{tabular}{llcc}
\hline Gene name & Domain name & Domain region (amino acid) & Amplification regions (amino acid) \\
\hline GEZE01034532 & TIR & $2-119$ & \\
& NB-ARC & $154-409$ & $268-680$ \\
GEZE01070617 & LRR & $433-928$ & \\
& CC & $2-126$ & $580-860$ \\
& NB-ARC & $168-463$ & \\
GEZE01020818 & LRR & $601-893$ & \\
& RPW8 & $9-126$ & $576-875$ \\
& NB-ARC & $185-452$ & \\
\hline
\end{tabular}

